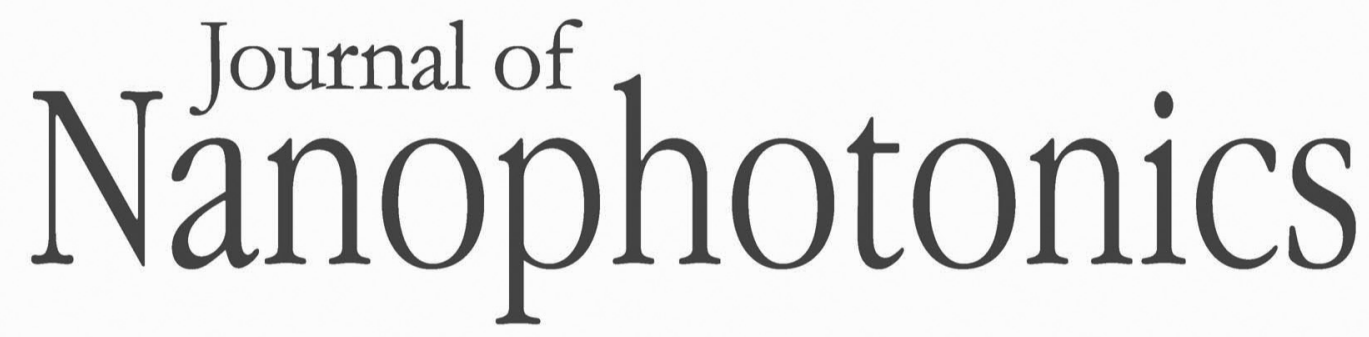

\title{
Special Section Guest Editorial: Nanobiophotonics and Related Techniques
}

Mun Seok Jeong 


\title{
Special Section Guest Editorial: Nanobiophotonics and Related Techniques
}

\author{
Mun Seok Jeong \\ Department of Energy Science, Center for Integrated Nanostructure Physics, \\ Sungkyunkwan University, Suwon 440-746, Republic of Korea \\ mjeong@skku.edu
}

Nanobiotechnology is the convergent field of nanotechnology and biotechnology, and it has received a lot of attention in the 21st century. Typical technical fields of nanobiotechnology are the design and synthesis of biological molecules with new functionality, nanobiosensors, nanoimaging technology of cell and biosystems, and nanomaterials for drug delivery and therapy. Nanotechnology based on the structure and principle of biomaterials is the emerging field to develop new nanodevices and systems that were not possible with existing macroscale technology. It is evaluated as a core technology that can lead to new industries in the 21 st century.

SPIE 2013 Nano-Bio Sensing, Imaging \& Spectroscopy (NBSIS) was the international conference on technologies and applications of nanobiosensing, nanobioimaging, and nanobiospectroscopy. It is quite obvious that nanobiotechnologies and applications are currently one of the most anticipated and challenging subjects to biologists as well as nanoscientists. This special section is composed of selected papers presented at NBSIS, and it will provide a useful overview to specialists in the field and engineers who will apply the technology.

\section{Acknowledgments}

The guest editor thanks the program committee and all authors for their contributions to this special section. 